\title{
Relación de la desalineación postural y la convergencia ocular con los trastornos temporomandibulares
}

\author{
Relationship of postural misalignment \\ and ocular convergence to \\ temporomandibular disorders
}

\section{Relação de desalinhamento postural e convergência ocular com desordens temporomandibulares}

\author{
Marcel Aguilar Salas, CD, MSC, PHD ${ }^{1 *}$ \\ Paola Ramos Luna, CD, MSC $^{2}$
}

Recibido: 26 de octubre de 2020 • Aceptado: 24 de mayo de 2021

Doi: https://doi.org/10.12804/revistas.urosario.edu.co/revsalud/a.9678

Para citar este artículo: Aguilar Salas M, Ramos Luna P. Relación de la desalineación postural y la convergencia ocular con los trastornos temporomandibulares . Rev Cienc Salud. 2021;19(2):1-15.

https://doi.org/10.12804/revistas.urosario.edu.co/revsalud/a.9678

\section{Resumen}

Introducción: los trastornos temporomandibulares son unas condiciones dolorosas o disfuncionales en los músculos masticadores o en la articulación temporomandibular con síntomas y signos complejos y variados que influyen negativamente en la calidad de vida. La evidencia científica es ambigua al querer relacionar la postura corporal con la presencia o ausencia de esta alteración. El objetivo es determinar la relación entre la postura de las cinturas escapular y pelviana y la convergencia ocular con los trastornos temporomandibulares. Materiales y métodos: se realizó una investigación observacional, correlacional, prospectiva, transversal. La población estaba constituida por 146 pacientes de 18 a 47 años que acudieron al servicio de odontología del Hospital Honorio Delgado Espinoza y que cumplieron con los criterios de elegibilidad. Se empleó el índice de Helkimo modificado por Maglione para la evaluación de los trastornos temporomandibulares, y para la postura de las cinturas escapular y pelviana y la convergencia ocular se empleó el protocolo de evaluación clínica del Dr. Dupas de la Universidad de Lille (Francia). Resultados: $91.1 \%$ presentaron trastornos temporomandibulares; $86.3 \%$, disfunción leve; $4.8 \%$, disfunción moderada, con un predominio femenino, en un 67.8\%. Las manifestaciones clínicas resultantes fueron desviación mandibular, ruidos articulares y limitación de movimiento mandibular

1 Universidad Católica de Santa María, Arequipa (Perú). oRcid https://orcid.org/0000-0001-6492-6126

2 Universidad Nacional deSan Agustín, Arequipa (Perú). orciD https://orcid.org/0000-0001-9523-7075

Autor de correspondencia: vaguilar@ucsm.edu.pe 
moderado. La relación entre la no alineación de las cinturas escapular y pelviana con los trastornos temporomandibulares fue altamente significativa y no se encontró relación con la convergencia ocular. Conclusiones: se evidenció una relación estadística altamente significativa entre los trastornos temporomandibulares y la postura alterada de las cinturas señaladas. No se encontró relación con la hipoconvergencia ocular.

Palabras clave: trastornos de la articulación temporomandibular; articulación temporomandibular; postura; posicionamiento del paciente; equilibrio postural; convergencia ocular.

\section{Abstract}

Introduction: Temporomandibular disorders are a set of painful and/or dysfunctional conditions of the chewing muscles and/or in the temporomandibular joint. They involve complex and varied symptoms and signs that negatively influence the quality of life. The scientific evidence is ambiguous regarding relating body posture with the presence or absence of the involved alterations. The objective of this study is to determine the relationship between the posture of the shoulder girdle, pelvic girdle, and ocular convergence with temporomandibular disorders. Materials and methods: An observational, correlational, prospective, cross-sectional investigation was conducted. The population consisted of 146 patients aged 18 to 47 years who attended the dental service of the Hospital Honorio Delgado Espinoza and met the eligibility criteria. The Helkimo index modified by Maglione was used for the evaluation of temporomandibular disorders. The posture of the shoulder girdle, pelvis, and ocular convergence were evaluated using the clinical evaluation protocol of Dr. Dupas of the University of Lille-France. Results: There was a total of $91.1 \%$ presented temporomandibular disorders. These included $86.3 \%$ with mild dysfunction and $4.8 \%$ with moderate dysfunction. There was a female predominance (67.8\%). The outstanding clinical manifestations were mandibular deviation, joint noises, and moderate mandibular movement limitation. The relationship between pelvic girdle and shoulder girdle nonalignment with temporomandibular disorders was highly significant. No relationship was found with ocular convergence. Conclusions: A highly significant statistical relationship was found between the temporomandibular disorders and the altered postures of the shoulder girdle and pelvis. No relationship was found with ocular hypoconvergence.

Keywords: Temporomandibular joint disorders; temporomandibular joint; posture; patient positioning; postural balance; convergence ocular.

\section{Resumo}

Introdução: as disfunções temporomandibulares são um conjunto de condições dolorosas ou disfuncionais dos músculos da mastigação ou da articulação temporomandibular com sintomas e sinais complexos e variados que influenciam negativamente na qualidade de vida. As evidências científicas são ambíguas ao relacionar a postura corporal com a presença ou ausência desta alteração. O objetivo é determinar a relação entre a postura da cintura escapular, cintura pélvica e convergência ocular com as disfunções temporomandibulares. Materiais e métodos: foi realizada uma investigação observacional, correlacional, prospectiva e transversal. A população foi constituída por 146 pacientes com idades entre 18 e 47 anos que frequentaram o serviço de odontologia do Hospital Honorio Delgado Espinoza e que preencheram os critérios de elegibilidade. O índice de Helkimo modificado por Maglione foi utilizado para a avaliação das disfunções temporomandibulares, já para a postura da cintura escapular, cintura pélvica e convergência ocular, foi utilizado o protocolo de avaliação clínica do Dr. Dupas da Universidade de Lille-França. Resultados: 91.1\% apresentavam disfunção temporomandibular, 86.3\% disfunção leve, 4.8\% disfunção moderada com predomínio do sexo feminino em $67.8 \%$. As manifestações clínicas marcantes foram desvio mandibular, ruídos articulares e limitação moderada dos movimentos mandibulares. A relação entre o não alinhamento da cintura escapular e da cintura pélvica com as disfunções temporomandibulares foi altamente significativa e nenhuma relação foi encontrada com a 
convergência ocular. Conclusões: foi evidenciada relação estatística altamente significativa entre disfunção temporomandibular e postura alterada da cintura escapular e cintura pélvica. Nenhuma relação foi encontrada com a hipoconvergência ocular.

Palavras-chave: disfunções da articulação temporomandibular; articulação temporomandibular; postura; posicionamento do paciente; equilíbrio postural; convergência ocular.

\section{Introducción}

L

os trastornos temporomandibulares (ттм) son un conjunto de condiciones dolorosas o disfuncionales en los músculos masticadores o en la articulación temporomandibular (ATM), con signos y síntomas variados como: limitación de la apertura bucal, dificultad masticatoria, dolor articular, dolor muscular, dolor de cabeza, migraña, dolor de oído, ruidos articulares, etc., y todo esto influye negativamente en la calidad del sueño y vida de las personas (1-3). El uso de planos o férulas desprogramadoras en busca de la relajación muscular ha mostrado una reducción significativa de la intensidad del dolor miofascial (4), pero deben considerarse muchos factores para poder tratarla completamente de manera más efectiva e integral. Se ha identificado la importancia del género en el desarrollo de los ттм, con un riesgo dos veces mayor de que las mujeres lo desarrollen en comparación con los varones $(5,6)$. También es importante considerar la postura corporal y de la cabeza en el diagnóstico y tratamiento de los ттм, pues existe una diferencia significativa en la estabilidad postural del cuerpo entre sujetos con тTм miogénica y controles sanos (7). Los adultos con dolor de cuello muestran una mayor postura de la cabeza hacia adelante en comparación con los adultos asintomáticos, y esta postura adelantada se correlaciona significativamente con las medidas de dolor de cuello en adultos y adultos mayores (8). Por lo tanto, la estabilidad ortostática del cráneo sobre la columna cervical está relacionada con los TTM y el dolor orofacial.

Es importante considerar que el estrés psicoemocional también tiene una repercusión significativa en la etiología de los ттм, pues muchas veces es el causante del efecto gatillo que desencadena un trastorno que permanecía asintomático $(4,9)$. La edad es otro factor por considerar: revisiones sistemáticas informan que los ттм tienen un pico de prevalencia entre los 25 y los 45 años y las estimaciones de necesidad de tratamiento evaluadas por metanálisis indican que para los sujetos más jóvenes, de 19 a 45 años, fueron más altas que para los sujetos de 46 años o más $(10,11)$.

Varias revisiones sistemáticas investigaron la relación entre la postura corporal, de la cabeza y cervical con los TTM, y se obtuvieron resultados controvertidos y poco claros, pero también hicieron hincapié en la mala calidad metodológica de dichos estudios $(12,13)$. 
El componente integral del cuadrante superior, básicamente formado por cabeza, cuello y cintura escapular, consta de numerosas estructuras esqueléticas, todas íntimamente relacionadas con articulaciones, uniones musculares, ligamentosas, etc. Las alteraciones posturales que originan hiperactividad muscular pueden variar la relación anatómica normal entre cabeza, cuello y cintura escapular, que con frecuencia resultan una importante causa de dolor y disfunción cráneo-mandibular (14).

Los músculos que conectan las cinturas escapular y pelviana (recto abdominal, gran dorsal, psoas iliaco, cuadrado lumbar, etc.) inducen una modificación de una sobre la otra y viceversa, que puede ser ipsolateral o contralateral. El estudio de la horizontalidad de la cintura escapular y la pelviana es la mejor y más simple forma de apreciar y evaluar la postura corporal (15).

Un problema ocular verdadero o el desequilibrio tónico postural inducido o no por la mandíbula repercute directamente o a través de la formación reticular sobre el III, iv y vi par craneal, que inerva los músculos oculomotores y alcanza una hipotonía muscular que se manifiesta en un problema de convergencia ocular (15).

El objetivo del presente artículo fue evaluar y determinar la relación entre la postura de la cintura escapular, la pelviana y la convergencia ocular con los ттм.

\section{Materiales y métodos}

S realizó una investigación observacional, descriptiva, prospectiva y transversal. La población estuvo constituida por 146 pacientes (99 mujeres y 47 varones) entre 18 y 47 años que acudieron a la consulta externa del servicio de odontología del Hospital Honorio Delgado Espinoza de la ciudad de Arequipa (Perú) entre agosto y noviembre de 2019 y que cumplieron con los siguientes criterios de inclusión: edad mayor a 18 años, ambos sexos, sin malformaciones o alteraciones músculo-esqueléticas; y exclusión: personas con odontalgia, otalgia, cefalea, algún tipo de parálisis muscular, fracturas anteriores o recientes en hombros, clavícula, brazos, caderas, invidentes y pacientes con ortodoncia.

Previo consentimiento firmado, se recolectaron los datos generales y clínicos de los pacientes incluidos en dos fichas que respetaron la normatividad del Comité de Ética del Hospital Honorio Delgado Espinoza, los principios éticos de la Declaración de Helsinki y la Ley General de Salud del Perú (Ley 268429). La información obtenida fue tratada de forma confidencial y manejada solo por las personas que participaron en la investigación.

Se identificaron los ттм y se determinó su prevalencia. Un solo observador (quien fue capacitado en la Universidad de Lille, Francia) evaluó la postura de las cinturas escapular y pelviana y la convergencia ocular. 
Para determinar la prevalencia de los Tтм se aplicó el Índice Clínico de Helkimo modificado por Maglione (16-18), por tener una mejor distribución tanto de puntuación en cada agrupación de síntomas y signos como una correspondencia más acentuada entre cada categoría de índice de severidad. El procedimiento consistió en la sumatoria de cinco manifestaciones clínicas: limitación del movimiento mandibular, alteración de la función articular, presencia de dolor al realizar algún movimiento mandibular, dolor muscular y dolor en la ATM. Cada una de ellas cubre tres posiciones posibles en cada caso: 0, 1 y 5 puntos. Así, se clasificaron de acuerdo con el puntaje obtenido en tres categorías ya establecidas: disfunción leve, moderada o severa.

Para evaluar la postura de las cinturas escapular y pelviana se aplicó el protocolo de evaluación clínica del Dr. P. Dupas de la Facultad de Odontología Universidad de Lille II, Francia (15). Este análisis se llevó cabo en un sillón dental que se encontraba con el espaldar reclinado a $180^{\circ}$. El paciente estaba recostado, centrado, con la piernas paralelas, relajadas y rectas. Los brazos permanecieron pegados al cuerpo hasta que el investigador tomó las muñecas del paciente y controló el nivel de los maléolos radiales.

La cintura escapular se evaluó por el largo de los brazos. Para ello el investigador se ubicó por detrás del sillón dental; tomó las muñecas del paciente entre el dedo pulgar, el índice y el medio, y tiró ligeramente los brazos de manera simétrica en el plano sagital. A fin de asegurarse de que la posición del paciente sobre el sillón no provocara algún error del test, se le pidió relajar los brazos. La maniobra debe ser lo suficientemente firme y rápida, y si la cintura escapular está horizontal, los maléolos radiales estarán paralelos (figura 1).

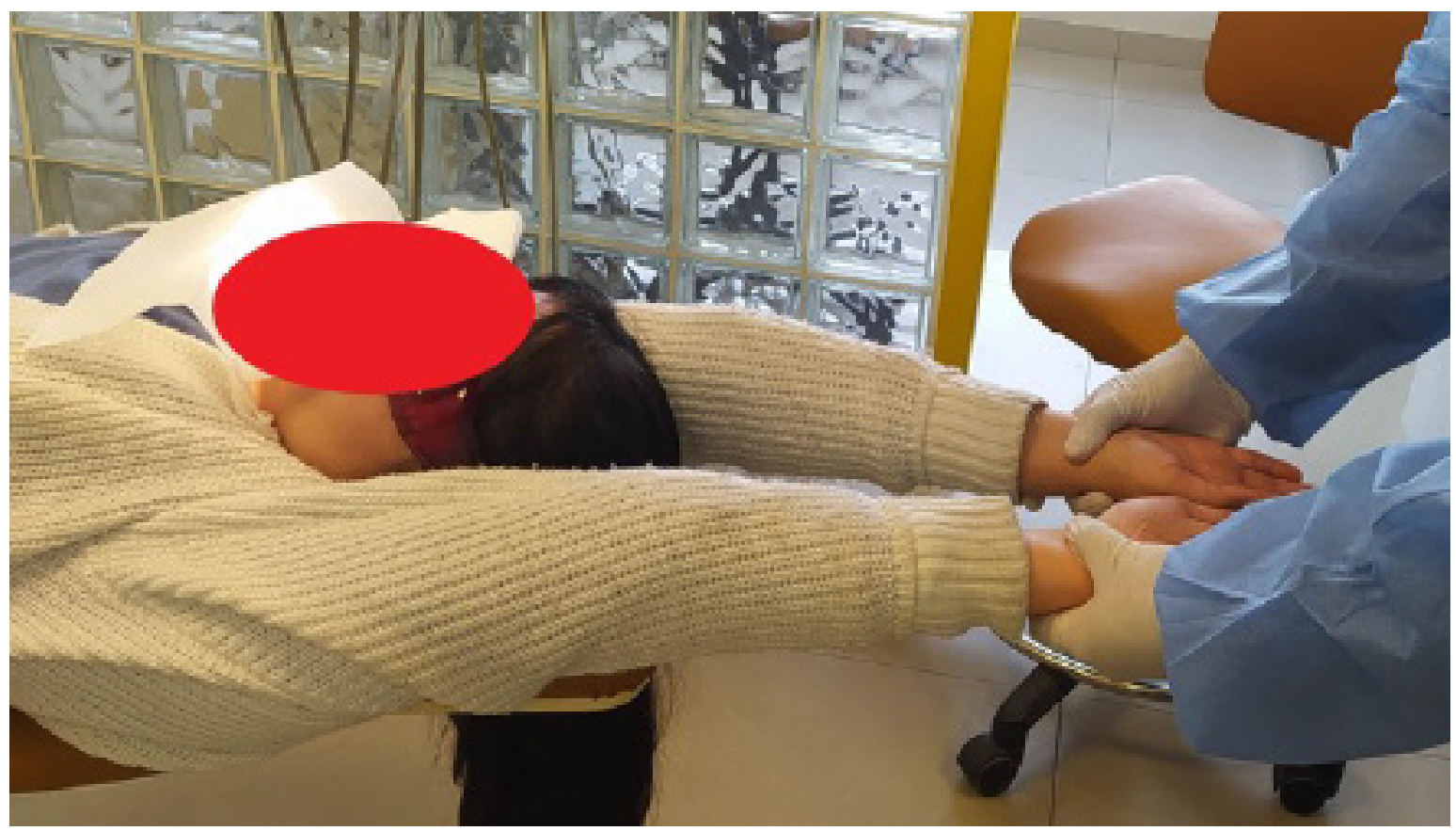

Figura 1. Evaluación de la cintura escapular 
Para la evaluación de la cintura pelviana en la misma posición de reclinación de $180^{\circ}$ del espaldar del sillón dental se verificó que el paciente estuviera cómodo, derecho, con las piernas paralelas y relajadas sin hacer tensión. El investigador se ubicó por delante de los pies y con los dedos de las maños rodeó los tobillos, ubicó los maléolos tibiales (parte más prominente) y verificó su posición para determinar si estaban paralelos o en el mismo nivel. Así se consideró alineada la cintura pelviana o, por el contrario, se consideró no alineada (figura 2). Ambas evaluaciones se realizaron con la boca entreabierta y sin contactos oclusales.

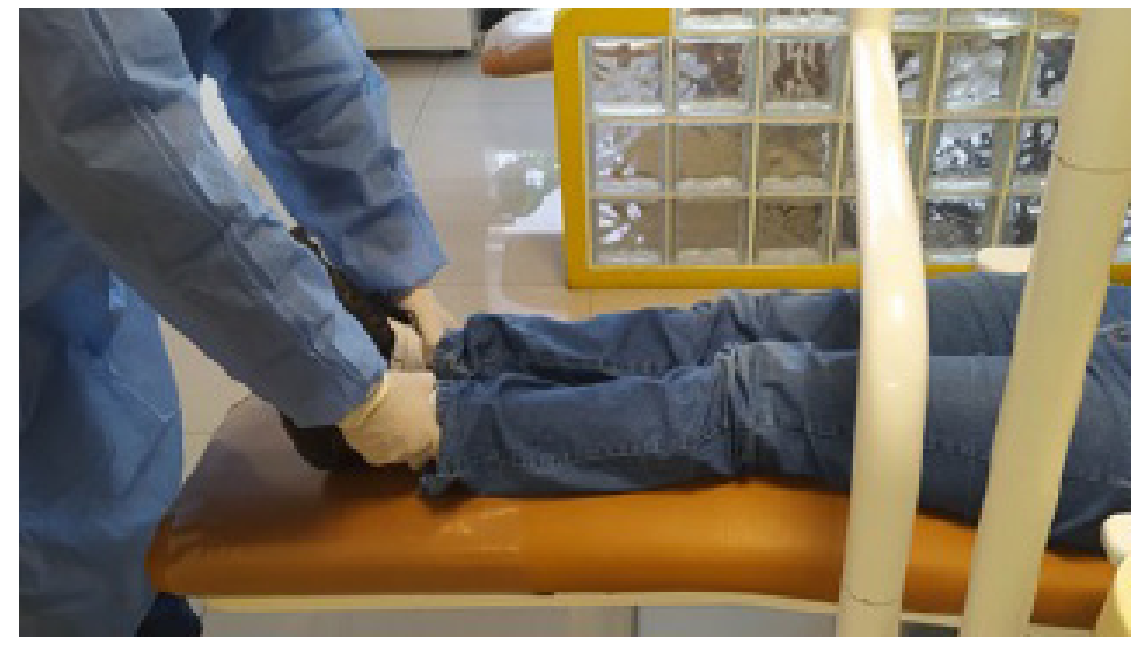

Figura 2. Evaluación de la cintura pelviana

Para analizar la convergencia ocular se aplicó el Test de Convergencia Ocular (15) en el consultorio dental, con luz natural opuesta a la visión del paciente. Este consiste en aproximar un lápiz, frente al paciente, a la altura de sus ojos, desde una distancia de $33 \mathrm{~cm}$ dentro del plano sagital. Así se le solicita al paciente seguir la punta del lápiz con los ojos. Se evaluaron y registraron los ojos de manera individual; así como el movimiento coordinado de los ojos para poder fijar el objeto que se aproximó; además, se consideró hipoconvergencia al desplazamiento no simétrico o no existente de cada ojo (figura 3). 


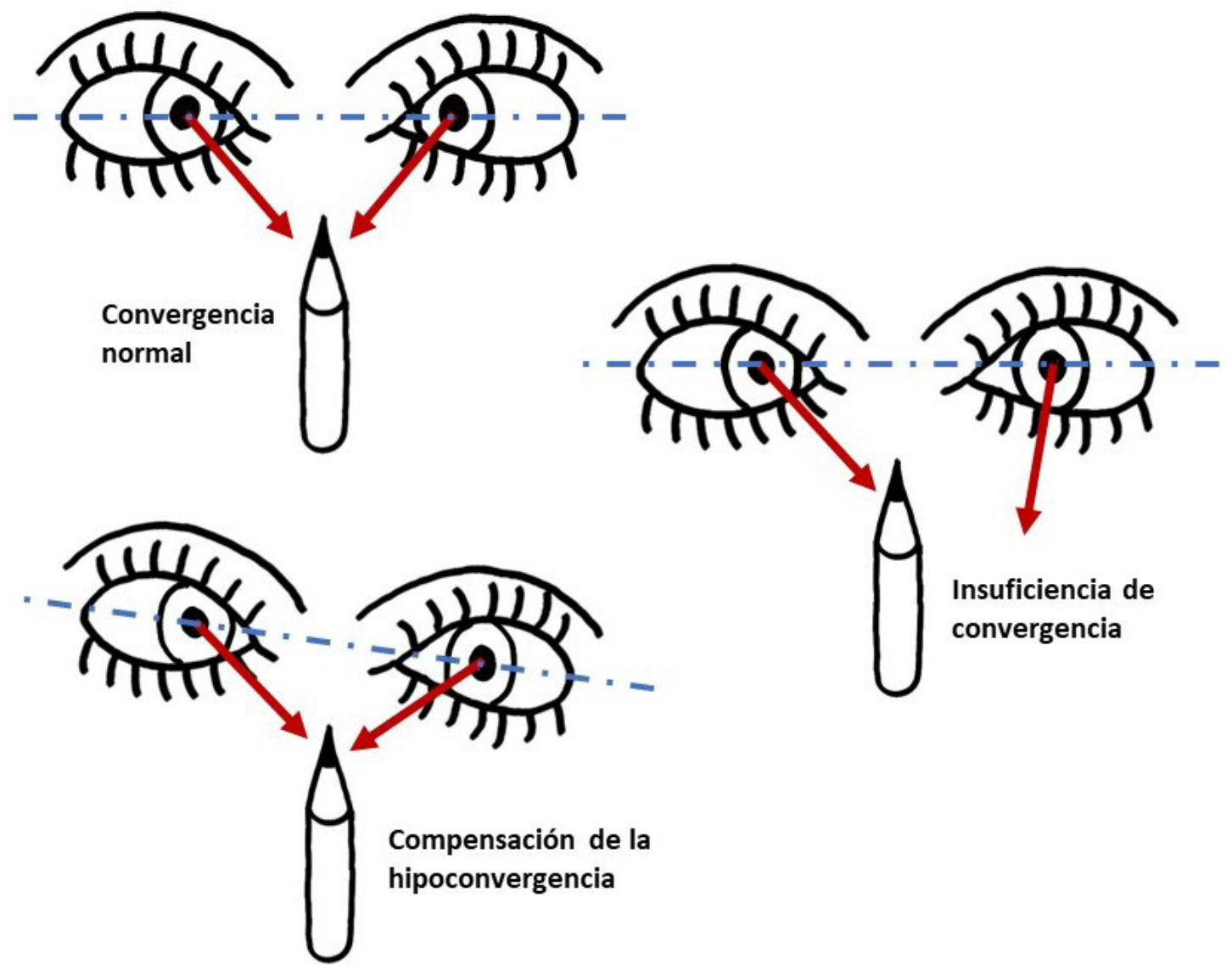

Figura 3. Evaluación de la convergencia ocular

Fuente: tomado de (15).

Los datos se ordenaron en una matriz de Excel y se procesaron con el software spss 25. Se empleó estadística descriptiva con frecuencias absolutas y porcentuales. Las relaciones entre los signos y síntomas de los ттм y cintura escapular, cintura pelviana y convergencia ocular se realizaron utilizando chi-cuadrado $\left(\chi^{2}\right)$ en un nivel de significación del $5 \%$ y con un coeficiente V de Cramer para evaluar la asociación entre variables.

\section{Resultados}

n el estudio se encontró que la moda es de 30 años y la mediana es de 29 años, con predo-minio del sexo femenino, en un $67.8 \%$. De acuerdo con el Índice de Helkimo modificado por Maglione, el $91.1 \%$ de los pacientes presentaron тTм; el $86.3 \%$ con disfunción leve y el $4.8 \%$ con disfunción moderada. Las mayores manifestaciones clínicas fueron desviación mandibular o presencia de ruidos articulares (55.5\%) y limitación de movimiento mandibular moderado (52.7\%). 
Hay relación entre la alineación de la cintura escapular y los тTм (tabla 1a), y entre la alineación de la cintura pelviana y los ттм (tabla 1b), según la prueba de $\chi^{2}$, con un $95 \%$ de confiabilidad, cuyos valores de significancia fueron de $p<0.05$ en ambos casos. Se reconoce una tendencia según la cual en тTм leves se presenta más desalineación escapular, y en тTM moderados, desalineación pelviana. Se aplicó la V de Cramer, de la cual se obtuvo un valor mayor a 0.2 , lo que indica una relación moderada entre variables.

Tabla 1a. Relación de los trastornos temporomandibulares con la alineación de cintura escapular

\begin{tabular}{|c|c|c|c|c|c|c|}
\hline \multirow{3}{*}{$\begin{array}{c}\text { Trastornos } \\
\text { temporomandibulares }\end{array}$} & \multicolumn{4}{|c|}{ Alineación de la cintura escapular } & \multirow{2}{*}{\multicolumn{2}{|c|}{ Total }} \\
\hline & \multicolumn{2}{|c|}{ No } & \multicolumn{2}{|c|}{ Sí } & & \\
\hline & $\mathbf{N}$ & $\%$ & $\mathbf{n}$ & $\%$ & $\mathbf{n}$ & $\%$ \\
\hline No presenta & 3 & 2.05 & 10 & 6.85 & 13 & 8.90 \\
\hline Leve & 78 & 53.42 & 48 & 32.88 & 126 & 86.30 \\
\hline Moderada & 7 & 4.79 & 0 & 0.00 & 7 & 4.79 \\
\hline Total & 88 & 60.27 & 58 & 39.73 & 146 & 100.00 \\
\hline
\end{tabular}

$\chi^{2}=12.266 ; p=0.002(p<0.01) ; \mathrm{V}$ de Cramer $=0.29$.

Tabla 1b. Relación de los trastornos temporomandibulares con la alineación de la cintura pelviana

\begin{tabular}{|c|c|c|c|c|c|c|}
\hline \multirow{3}{*}{$\begin{array}{c}\text { Trastornos } \\
\text { temporomandibulares }\end{array}$} & \multicolumn{4}{|c|}{ Alineación de la cintura pelviana } & \multirow{2}{*}{\multicolumn{2}{|c|}{ Total }} \\
\hline & \multicolumn{2}{|c|}{ No } & \multicolumn{2}{|c|}{ Sí } & & \\
\hline & $\mathbf{n}$ & $\%$ & $\mathbf{n}$ & $\%$ & $\mathbf{n}$ & $\%$ \\
\hline No presenta & 0 & 0.00 & 13 & 8.90 & 13 & 8.90 \\
\hline Leve & 35 & 23.97 & 91 & 62.33 & 126 & 86.30 \\
\hline Moderada & 5 & 3.42 & 2 & 1.37 & 7 & 4.79 \\
\hline Total & 40 & 27.40 & 106 & 72.60 & 146 & 100.00 \\
\hline
\end{tabular}

$\chi^{2}=11.738 ; p=0.003(p<0.01) ; \mathrm{V}$ de Cramer $=0.284$

Al analizar la hipoconvergencia ocular obtenida en los ojos derecho e izquierdo, esta fue del $8.22 \%$ y del $15.07 \%$, respectivamente. No se encontró mayor predominio. Así, no se encontró una relación estadísticamente significativa entre convergencia ocular y ттм (tablas 2a y 2b), según la prueba de $\chi^{2}$, con un $95 \%$ de confiabilidad, cuyos valores de significancia fueron $p>0.05$ en ambos casos. Se aplicó la V de Cramer, de la cual se obtuvo un valor menor a 0.2, que indica que es despreciable la relación entre las variables. 
Tabla 2a. Relación de los trastornos temporomandibulares con la hipoconvergencia ocular del ojo derecho

\begin{tabular}{|c|c|c|c|c|c|c|}
\hline \multirow{3}{*}{$\begin{array}{c}\text { Trastornos } \\
\text { temporomandibulares }\end{array}$} & \multicolumn{4}{|c|}{ Hipoconvergencia ocular del ojo derecho } & \multirow{2}{*}{\multicolumn{2}{|c|}{ Total }} \\
\hline & \multicolumn{2}{|c|}{ Sí } & \multicolumn{2}{|c|}{ No } & & \\
\hline & $\mathbf{N}$ & $\%$ & $\mathbf{n}$ & $\%$ & $\mathbf{n}$ & $\%$ \\
\hline No presenta & 1 & 0.68 & 12 & 8.22 & 13 & 8.90 \\
\hline Leve & 10 & 6.85 & 116 & 79.45 & 126 & 86.30 \\
\hline Moderada & 1 & 0.68 & 6 & 4.11 & 7 & 4.79 \\
\hline Total & 12 & 8.22 & 134 & 91.78 & 146 & 100.00 \\
\hline
\end{tabular}

$\chi^{2}=0.360 ; p=0.835(p>0.01) ; V$ de Cramer $=0.05$.

Tabla 2b. Relación de los trastornos temporomandibulares con la hipoconvergencia ocular del ojo izquierdo

\begin{tabular}{|c|c|c|c|c|c|c|}
\hline \multirow{3}{*}{$\begin{array}{c}\text { Trastornos } \\
\text { Temporomandibulares }\end{array}$} & \multicolumn{4}{|c|}{ Hipoconvergencia ocular del ojo izquierdo } & \multirow{2}{*}{\multicolumn{2}{|c|}{ Total }} \\
\hline & \multicolumn{2}{|c|}{ Sí } & \multicolumn{2}{|c|}{ No } & & \\
\hline & $\mathbf{N}$ & $\%$ & $\mathbf{n}$ & $\%$ & $\mathbf{n}$ & $\%$ \\
\hline No presenta & 0 & 0.00 & 13 & 8.90 & 13 & 8.90 \\
\hline Leve & 20 & 13.70 & 106 & 72.60 & 126 & 86.30 \\
\hline Moderada & 2 & 1.37 & 5 & 3.42 & 7 & 4.79 \\
\hline Total & 22 & 15.07 & 124 & 84.93 & 146 & 100.00 \\
\hline
\end{tabular}

$\chi^{2}=3.367 ; p=0.186(p>0.01) ; \mathrm{V}$ de Cramer $=0.152$.

\section{Discusión}

$\mathrm{D}$ esde 1934, cuando el Dr. James Costen describió los síntomas referidos al oído y la ATM, se inició una constante búsqueda e investigación de las causas intrínsecas y extrínsecas que produzcan un ттм. A través de estos años y por los estudios llevados a cabo, se demostró que la frecuencia es alta y la disminución de esta también, conforme se conoce más, como lo demostraron Gil-Martínez et al. (6), quienes indicaron que el ттм es un problema muy frecuente que puede tratarse de manera invasiva y no invasiva. Cantero et al. indicaron que aproximadamente entre el $70 \%$ y el $90 \%$ de la población general tiene, al menos, un signo clínico de disfunción (17). Espinosa de Santillana et al. describieron las alteraciones posturales más frecuentes según el tipo de ттм identificado: la mayor frecuencia de ттм, el tipo articular, seguida de la muscular y, al final, combinado (19). Así, las alteraciones posturales más comunes fueron: hombro alto, inclinación pélvica, postura de la cabeza hacia adelante, y de estos el $100.0 \%$ de los pacientes tenían alteraciones en la vista lateral. Concluyeron que los pacientes con ттм tienen cambios posturales. Sousa et al. evaluaron la relación entre la postura corporal 
y los ттм y concluyeron que los sujetos con ттм y sin estos presentaron una postura corporal global desalineada, pero estos cambios fueron más pronunciados en pacientes con тTм (20).

Al-Khotani et al., Bertoli et al. y Lövgren et al. informaron que la prevalencia de тTм es del $27.2 \%$, 34.9\% y $30 \%$, respectivamente, valores muy diferentes al encontrado en la presente investigación: 91.1\% (21-23). Esto se debe a la población estudiada: los primeros son niños, adolescentes y estudiantes de odontología sanos; los segundos son pacientes que acuden a un servicio público de odontología de un hospital de referencia. Los тTM podrían ser más frecuentes en personas de mayor edad, porque están asociados con parafunciones, detonadas muchas veces por el estrés emocional, que aumenta la tonicidad muscular e incrementa la actividad muscular parafuncional. Dentro de ellos, principalmente el bruxismo, que constituye otro mecanismo de descarga interna de estrés, como lo corroboraron Huhtela et al., El Hage et al. (24,25). Estos datos no se corresponden con los encontrados en la investigación, pero sí con los informados por Ryan et al. y Al-Jundi et al., quienes informan una mayor frecuencia en el grupo de 19 a 45 años, aproximadamente $(10,11)$.

En cuanto a las manifestaciones clínicas, se observa que un 56\% presenta desviación mandibular o presencia de ruidos articulares durante el movimiento de apertura y un $50 \%$ presenta un moderado deterioro de la movilidad mandibular. Estos resultados se asemejan a los encontrados por Mapelli et al., quienes indican que los movimientos de abertura, cierre y lateralidad son significativamente más lentos en los pacientes con disfunción (26).

Los ттм deben evaluarse de manera integral considerando sus diferentes manifestaciones y no centrarse solamente en las estructuras anatómicas. Pihut et al. demostraron que la forma de dolor de los тTM no estaba relacionada con la inflamación de las ATM; es por ello por lo que también se relacionó con la postura corporal (27). Sánchez et al. señalaron que los pacientes con disfunción presentaban, sobre todo, dolor muscular temporomandibular si exhibían alteraciones de la postura (28). Montero et al. hallaron que los pacientes con chasquidos de ATM tenían la pelvis y hombros desequilibrados en el mismo sentido, pero contrario al lugar donde aparecía el signo (29). Según Fuentes et al., existe una relación estadísticamente significativa entre la alteración de la postura de las caderas y la prevalencia de тTM, así como entre las alteraciones de la postura de los hombros y la prevalencia de тTM (30). En la presente investigación se obtuvieron resultados similares y con valores estadísticamente significativos. Sin embargo, investigaciones que se dirigieron más a evaluar el sistema esquelético no lograron mayor relación, como Raya et al., que no encontraron una relación significativa entre la disfunción craneomandibular y alteraciones cervicales, o Lunes et al., quienes concluyeron que la postura de la cabeza y columna cervical no diferían entre el grupo con ттм o sin estos (31,32).

La alineación postural podría entonces orientarse más al estado muscular, como lo evaluado por Fuentes et al. (30), quienes encontraron una relación significativa entre el lado del cuerpo en que el hombro o la cadera estaba más baja y con mayor sensibilidad a la 
palpación de la ATM, o Giacomo et al., quienes informaron que el dolor más común fue en la correspondencia de los puntos de la ATM y el músculo temporal, los músculos pterigoideos externos y los maseteros con intensidad severa y frecuencia crónica $(30,33)$.

Desde hace varios años se han estudiado las posibles relaciones que existirían entre la oftalmología y el sistema estomatognático. En la presente investigación no se encontró relación alguna entre la hipoconvergencia ocular y los ттм, los cuales contrastan con los encontrados por Cuccia et al., quienes descubrieron que los pacientes que sufren trastornos internos de ATM mostraron una convergencia reducida, en comparación con los sujetos sanos (34). Marchili et al. demostraron la relación entre maloclusiones, ттм y defectos visuales, y destacaron una mayor prevalencia de miopía en pacientes con maloclusiones de clase ir que en pacientes con maloclusiones de clase Iy III, así como una mayor prevalencia de pacientes con astigmatismo (35). El Hage et al. hallaron que el efecto de las alteraciones en los músculos de la masticación y los ligamentos dentoalveolares en el sistema estomatognático puede llevar a un trastorno de la estabilización visual y llevar a un desequilibrio postural (25). Baldini et al. determinaron que la visión ejerce un papel central en el sistema postural en los pilotos de la fuerza aérea y los pilotos civiles, lo cual indica que la función visual permite un mejor control postural (36). La evaluación de la convergencia ocular en el presente trabajo fue realizada en el sillón dental.

Los resultados de esta investigación evidencian una relación estadísticamente significativa entre los ттм y la postura alterada de la cintura escapular y pelviana. No hubo relación con la hipoconvergencia ocular según el método de Helkimo modificado por Maglione y los protocolos de evaluación clínica del Dr. P. Dupas en pacientes del servicio de odontología del Hospital Honorio Delgado Espinoza. Conocer de qué manera se relacionan los тTM con la desalineación postural les permitirá a los profesionales comprender mejor los fenómenos que conducen a esta patología, lograr un diagnóstico más asertivo y ofrecer un tratamiento integral más adecuado, en busca de la mejora de la calidad de vida del paciente y el alivio del dolor.

\section{Contribución de los autores}

$\mathrm{M}$ arcel Aguilar Salas se encargó del diseño, concepción del estudio, análisis e interpretación de resultados, redacción, revisión crítica sustancial del contenido intelectual y revisión final del artículo que se iba a publicar. Paola Ramos Luna se encargó del diseño, concepción del estudio, recolección, análisis e interpretación de resultados, redacción y revisión final del artículo que se iba a publicar. 


\section{Conflicto de intereses}

Ninguno declarado.

\section{Descargos de responsabilidad}

工cial de las instituciones. Se declara que el financiamiento de esta investigación se realizó con fondos propios de los autores.

\section{Referencias}

1. Ferreira MC, Porto de Toledo I, Dutra KL, Stefani FM, Porporatti AL, Flores-Mir C, et al. Association between chewing dysfunctions and temporomandibular disorders: A systematic review. J Oral Rehabil. 2018;45(10):819-35. https://doi.org/10.1111/joor.12681

2. Oliveira LK, Almeida G de A, Lelis ÉR, Tavares M, Fernandes Neto AJ. Temporomandibular disorder and anxiety, quality of sleep, and quality of life in nursing professionals. Braz Oral Res. 2015;29(1):1-7. https://doi.org/10.1590/1807-3107bor-2015.vol29.0070

3. Bitiniene D, Zamaliauskiene R, Kubilius R, Leketas M, Gailius T, Smirnovaite K. Quality of life in patients with temporomandibular disorders: a systematic review. Stomatologija [internet]. 2018;20(1):3-9. https://sbdmj.lsmuni.lt/181/181-01.pdf

4. Ferendiuk E, Biegańska JM, Kazana P, Pihut M. Progressive muscle relaxation according to Jacobson in treatment of the patients with temporomandibular joint disorders. Folia Med Cracov. 2019;59(3):113-22. https://doi.org/10.24425/fmc.2019.131140

5. Bueno CH, Pereira DD, Pattussi MP, Grossi PK, Grossi ML. Gender differences in temporomandibular disorders in adult populational studies: a systematic review and meta-analysis. J Oral Rehabil. 2018;45(9):720-9. https://doi.org/10.1111/joor.12661

6. Gil-Martínez A, Paris-Alemany A, López-de-Uralde-Villanueva I, La Touche R. Management of pain in patients with temporomandibular disorder (TMD): challenges and solutions. J Pain Res. 2018;11:571-87. https://doi.org/10.2147/jpr.s127950

7. Nota A, Tecco S, Ehsani S, Padulo J, Baldini A. Postural stability in subjects with temporomandibular disorders and healthy controls: A comparative assessment. J Electromyogr Kinesiol. 2017;37:21-4. https://doi.org/10.1016/j.jelekin.2017.08.006

8. Mahmoud NF, Hassan KA, Abdelmajeed SF, Moustafa IM, Silva AG. The relationship between forward head posture and neck pain: a systematic review and meta-analysis. Curr Rev Musculoskelet Med. 2019;12(4):562-77. https://doi.org/10.1007/s12178-019-09594-y

9. Poveda Roda R, Bagan JV, Díaz Fernández JM, Hernández Bazán S, Jiménez Soriano Y. Review of temporomandibular joint pathology. Part I: classification, epidemiology and 
risk factors. Med Oral Patol Oral Cir Bucal [internet]. 2007;12(4):E292-298. http://www. medicinaoral.com/pubmed/medoralv12_i4_pE292.pdf

10. Ryan J, Hassan N, Hilton G, Wickham J, Akhter R, Ibaragi S. Epidemiology of Temporomandibular Disorder in the General Population: a Systematic Review. ADOH. 2019;10(3):1-13. https://doi.org/10.19080/ADOH.2019.10.555787

11. Al-Jundi MA, John MT, Setz JM, Szentpétery A, Kuss O. Meta-analysis of treatment need for temporomandibular disorders in adult nonpatients. J Orofac Pain. 2008;22(2):97-107.

12. 12. Rocha CP, Croci CS, Caria PHF. Is there relationship between temporomandibular disorders and head and cervical posture? A systematic review. J Oral Rehabil. 2013;40(11):87581. https://doi.org/10.1111/joor.12104

13. Chaves TC, Turci AM, Pinheiro CF, Sousa LM, Grossi DB. Static body postural misalignment in individuals with temporomandibular disorders: a systematic review. Braz J Phys Ther. 2014;18(6):481-501. https://doi.org/10.1590/bjpt-rbf.2014.0061

14. Ricard F. Tratado de osteopatía craneal. Articulación temporomandibular: análisis y

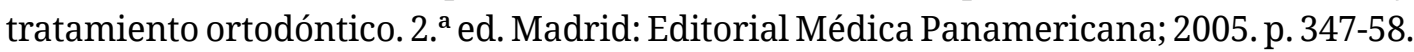

15. Dupas PH. Nouvelle approche du dysfonctionnement cranio-mandibulaire: du diagnostic à la gouttière. Paris: Cahiers de prothèses éditions; 2005. p. 97-131.

16. Santana MR, Moreno Chala Y, Rosales Rosales K, Osorio Capote Y, Morales Cordoví L. Grado de disfunción temporomandibular en mayores de 19 años. MEDISAN [internet]. 2013;17(12):9087-93. Disponible en: http://scielo.sld.cu/scielo.php?script=sci_arttext\&pi$\mathrm{d}=\mathrm{S} 1029-30192013001200009$

17. Cantero LS, Morales JD de la T, Espinosa IA, Rodríguez E de la T. Trastornos temporomandibulares en pacientes con maloclusiones. Rev Cubana Estomatol [internet]. 2013;50(4):20-6. Disponible en: http://www.revestomatologia.sld.cu/index.php/est/article/view/229

18. Alvarado Menacho S. Importancia de los índices simplificados en el diagnóstico y estudio de los trastornos temporomandibulares. Rev Estomatol Herediana. 2019 EneMar;29:89-94. https://doi.org/10.20453/reh.v29i1.3498

19. Espinosa de Santillana IA, García-Juárez A, Rebollo-Vázquez J, Ustarán-Aquino AK. Frequent postural alterations in patients with different types of temporomandibular disorders. Rev Salud Pública (Bogotá). 2018;20(3):384-9. https://doi.org/10.15446/rsap. v20n3.53529

20. Souza JA, Pasinato F, Corrêa ECR, da Silva AMT. Global body posture and plantar pressure distribution in individuals with and without temporomandibular disorder: a preliminary study. J Manipulative Physiol Ther. 2014;37(6):407-14. https://doi.org/10.1016/j. jmpt.2014.04.003

21. Al-Khotani A, Naimi-Akbar A, Albadawi E, Ernberg M, Hedenberg-Magnusson B, Christidis N. Prevalence of diagnosed temporomandibular disorders among Saudi Arabian children and adolescents. J Headache Pain. 2016;17:41. https://doi.org/10.1186/ s10194-016-0642-9

22. Bertoli FM de P, Bruzamolin CD, Pizzatto E, Losso EM, Brancher JA, de Souza JF. Prevalence of diagnosed temporomandibular disorders: A cross-sectional study in Brazilian adolescents. Plos One. 2018;13(2):e0192254. https://doi.org/10.1371/journal.pone.0192254 
23. Lövgren A, Österlund C, Ilgunas A, Lampa E, Hellström F. A high prevalence of TMD is related to somatic awareness and pain intensity among healthy dental students. Acta Odontol Scand. 2018;76(6):387-93. https://doi.org/10.1080/00016357.2018.1440322

24. Huhtela O, Näpänkangas R, Joensuu T, Raustia A, Kunttu K, Sipilä K. Self-Reported Bruxism and Symptoms of Temporomandibular Disorders in Finnish University Students. J Oral Facial Pain Headache. 2016;30(4):311-7. https://doi.org/10.11607/ofph.1674

25. El Hage Y, Politti F, de Sousa DFM, Herpich CM, Gloria IP dos S, Gomes CAF de P, et al. Effect of mandibular mobilization on electromyographic signals in muscles of mastication and static balance in individuals with temporomandibular disorder: study protocol for a randomized controlled trial. Trials. 2013;14:316. https://doi.org/10.1186/1745-6215-14-316

26. Mapelli A, Machado BCZ, Garcia DM, Rodrigues Da Silva M a. M, Sforza C, de Felício $\mathrm{CM}$. Three-dimensional analysis of jaw kinematic alterations in patients with chronic TMD - disc displacement with reduction. J Oral Rehabil. 2016;43(11):824-32. https://doi. org/10.1111/joor.12424

27. Pihut M, Ceranowicz P, Gala A. Evaluation of C-Reactive Protein Level in Patients with Pain Form of Temporomandibular Joint Dysfunction. Pain Res Manag. 2018;2018. https:// doi.org/10.1155\%2F2018\%2F7958034

28. Julià-Sánchez S, Álvarez-Herms J, Gatterer H, Burtscher M, Pagès T, Viscor G. The influence of dental occlusion on the body balance in unstable platform increases after high intensity exercise. Neurosci Lett. 2016;617:116-21. https://doi.org/10.1016/j.neulet.2016.02.003

29. Montero Parrilla JM, Semykina O, Morais Chipombela L da C. Trastornos temporomandibulares y su interacción con la postura corporal. Rev Cubana Estomatol [internet]. 2014;51(1):3-9. Disponible en: http://www.revestomatologia.sld.cu/index.php/est/article/ view/42

30. Fuentes R, Freesmeyer W, Henríquez J. Influencia de la postura corporal en la prevalencia de las disfunciones craneomandibulares. Rev Med Chil. 1999 Sep;127(9):1079-85. https://doi.org/10.4067/S0034-98871999000900007

31. Raya CR, Plaza-Manzano G, Pecos-Martín D, Ferragut-Garcías A, Martín-Casas P, GallegoIzquierdo T, et al. Role of upper cervical spine in temporomandibular disorders. J Back Musculoskelet Rehabil. 2017;30(6):1245-50. https://doi.org/10.3233/bmr-169620

32. Lunes DH, Carvalho LCF, Oliveira AS, Bevilaqua-Grossi D. Craniocervical posture analysis in patients with temporomandibular disorder. Braz J Phys Ther. 2009;13(1):89-95. https://doi.org/10.1590/S1413-35552009005000011

33. Di Giacomo P, Celli M, Ierardo G, Polimeni A, Di Paolo C. Evaluation of temporomandibular disorders and comorbidities in patients with Ehler-Danlos: clinical and digital findings. J Int Soc Prev Community Dent. 2018;8(4):333-8. https://doi.org/10.4103/jispcd. jispcd_103_18

34. Cuccia A, Caradonna C. The relationship between the stomatognathic system and body posture. Clinics (Sao Paulo). 2009;64(1):61-6. https://doi.org/10.1590/s180759322009000100011 
Evaluación de las cinturas escapular-pelviana y convergencia ocular con los trastornos temporomandibulares

35. Marchili N, Ortu E, Pietropaoli D, Cattaneo R, Monaco A. Dental occlusion and ophthalmology: a literature review. Open Dent J. 2016;10:460-8. https://doi.org/10.2174/187421 0601610010460

36. Baldini A, Nota A, Caruso S, Tecco S. Correlations between the Visual Apparatus and Dental Occlusion: A Literature Review. Biomed Res Int. 2018;07:1-12. https://doi. org/10.1155/2018/2694517 